\title{
A AGÊNCIA NACIONAL DE VIGILÂNCIA SANITÁRIA E A PESQUISA CLÍNICA NO BRASIL
}

Sérgio de Andrade Nishioka*, Paula Frassineti Guimarães de Sá

Trabalho realizado na gerência de Medicamentos Novos, Pesquisa e Ensaios Clínicos, da Agência Nacional de Vigilância Sanitária- Anvisa, Brasília, DF.

\author{
* Correspondência: \\ Agência Nacional de \\ Vigilância Sanitária, \\ Gerência de Medicamentos \\ Novos, Pesquisa e Ensaios \\ Clínicos \\ SEPN 515, Bloco B, \\ Edifício Omega, $2^{\circ}$ andar, \\ Sala 6, Brasília, DF, \\ Cep: 70770-502, \\ Fone: (61) 3448-1209. \\ sergio.nishioka@anvisa.gov.br
}

\begin{abstract}
RESUMO
A realização de ensaios clínicos com medicamentos no Brasil requer aprovação prévia pelos Comitês de Ética em Pesquisa (CEPs) e, em certos casos, pela Comissão Nacional de Ética em Pesquisa (CONEP). Aprovação pela Agência Nacional de Vigilância Sanitária (ANVISA), através de sua Gerência de Medicamentos Novos, Pesquisa e Ensaios Clinicos (GEPEC) é também necessária para ensaios clínicos com medicamentos e produtos para a saúde (correlatos) que são fabricados em outros páises e, portanto, necessitam autorizaçãa para serem importados. Este artigo revê brevemente a história da legislação sobre pesquisa clínica no Brasil, e apresenta um panorama do papel regulador da ANVISA no presente e suas perspectivas futuras. Pontos importantes discutidos são as legislações novas e futuras da ANVISA, com ênfase nas notificações de eventos adversos e inspeçōes/auditorias em ensaios clínicos, e seu impacto na pesquisa clínica no Brasil sob o ponto de vista da indústria, da universidade, organizaç̧ões representativas de pesquisa clínica, médicos e outros profissionais de saúde. A importância para a ANVISA da obtenção de informaç̧̃es a partir de ensaios clínicos para a avaliação do registro de novos medicamentos também é ressaltada.
\end{abstract}

UnITERMOS: ANVISA. Ensaio clínico. Legislação. Pesquisa clínica.

\section{INTRODUÇÃo}

A pesquisa clínica no Brasil vem apresentando um grande crescimento nos últimos dez anos. Por motivos históricos, para se realizar ensaios clínicos com medicamentos neste país faz-se necessária, particularmente quando se quer estudar produtos fabricados no exterior, a aprovação do protocolo de pesquisa por dois órgãos governamentais, a Comissão Nacional de Ética em Pesquisa (CONEP), que é ligada ao Conselho Nacional de Saúde (CNS), e a Agência Nacional de Vigilância Sanitária (ANVISA). Esta necessidade de dois fóruns de aprovação é confusa para muitos investigadores e patrocinadores de ensaios clínicos. Neste artigo, após um breve histórico da legislação sobre pesquisa clínica no Brasil, discute-se a visão da ANVISA quanto ao seu papel regulador dos ensaios clínicos, e enumera-se as medidas que vêm sendo propostas para regulamentá-la.

\section{Histórico}

A primeira tentativa de regulamentar a pesquisa em problemas de saúde no Brasil por meio de legislação espećífica foi em 1988, com a resolução $n^{\circ}$ I do Conselho Nacional de Saúde (CNS) ',2, considerando que a legislação vigente até então regulamentava somente alguns aspectos relacionados à importação de drogas destinadas exclusivamente à pesquisa, não registradas no país (Lei n $6.360 / 76$ e Decreto $n^{\circ} 79.094 / 77$ ). Esta resolução teve um impacto mínimo, e só a partir de 1996, com a publicação da Resolução CNS n. 196/96 e outras (particularmente a Resolução CNS n⿳. 25I/97) que a complementam é que começou a se consolidar uma legislação brasileira sobre pesquisa clínica². Tais resoluções, além de caracterizarem um compromisso das autoridades governamentais com os voluntários de pesquisa, representaram um passo significativo para a criação de indumentárias legais essenciais para a regulamentação da pesquisa clínica no Brasil, pois por meio da estruturação dos Comitês de Ética em Pesquisa (CEPs) nas diversas instituições de pesquisa e da constituiç̧ão da Comissão Nacional de Ética em Pesquisa (CONEP) explicitou-se a preocupação do Estado com a segurança e a garantia de que os direitos do sujeito de pesquisa fossem respeitados durante a condução dos ensaios clínicos. A reboque dessas resoluções do CNS, surgiu a primeira legislação sanitária sobre pesquisa clínica, a Portaria n 9 | I da Secretaria Nacional de Vigilância Sanitária (SVS), publicada em 1998 e ainda vigente, a qual, contemplando as atividades previstas pela Lei $n^{\circ} 6360 / 76$, lista os documentos e procedimentos necessários para a aprovação de protocolos de ensaios clínicos no Brasil. Com a criação da ANVISA, em 1999 (Lei n ${ }^{\circ}$ 9782/99), esta passou a substituir a SVS no papel de regulação sanitária dos ensaios clínicos no Brasil, sendo a anuência e monitorizaçãa dos mesmos atribuídas à Gerência de Medicamentos Novos, Pesquisa e Ensaios Clínicos (GEPEC).

\section{O papel da ANVISA na regulação da pesquisa clínica: presente}

Das competências atribuídas à GEPEC, destaca-se a análise e emissão de pareceres conclusivos nos processos referentes ao registro de medicamentos novos, bem como a autorização de projetos de pesquisa clínica a serem conduzidos em território nacional. Vale destacar as peculiaridades da sistematização da análise dos dossiês de pesquisa clínica na GEPEC, considerando que ela ocorre somente após eles terem sido aprovados pelos Comitês de Ética em Pesquisa (CEPs), que os avaliam em nível local, e é feita em paralelo com a CONEP, sempre que se faça necessária a avaliação por esta comissão, como é o caso da grande maioria dos ensaios clínicos patrocinados do exterior. 
É importante salientar que a Portaria $n^{\circ}$ 911/98 abrange todos os estudos conduzidos com quaisquer produtos sob vigilância sanitária (medicamentos, produtos para a saúde, cosméticos, saneantes, alimentos), mas, na prática, a imensa maioria dos estudos avaliados pela GEPEC são protocolos de pesquisa com medicamentos e, em menor escala, produtos para a saúde, anteriormente conhecidos como correlatos. Contudo, é possível observar que esta portaria é muito limitada em seu escopo, já que a sua finalidade básica é a de permitir que os produtos sob investigação nos ensaios clínicos possam ser legalmente importados, o que é feito por meio da concessão, pela GEPEC, de um comunicado especial (CE) que permite a emissão do licenciamento de importação (LI). Assim sendo, ensaios clínicos realizados com medicamentos (e produtos para a saúde) desenvolvidos no Brasil, ou já aqui registrados e comercializados, atualmente não requerem aprovação da ANVISA, e não são avaliados por esta agência. Os protocolos submetidos para a GEPEC são avaliados, mas além da comprovação da apresentação do protocolo e dos demais documentos exigidos pela Portaria $n^{\circ}$ 91 I/98 exige-se basicamente o cumprimento das resoluções do CNS. Como a aprovação dos protocolos de pesquisa não está ligada diretamente à sua utilização como evidência de eficácia e segurança para o registro do produto, a avaliação da GEPEC não tem tido esse olhar, até porque não há respaldo legal para que isso seja feito.

A ANVISA vê com bons olhos que ensaios clínicos com novos medicamentos, financiados do exterior, sejam desenvolvidos no Brasil. Além de oferecer informações sobre eficácia e segurança desses produtos nas condições locais e em nossa população, informações essas úteis e necessárias no processo de registro, a realização de tais estudos propicia treinamento a investigadores brasileiros e campo de trabalho para profissionais graduados em ciências da saúde. É de interesse da ANVISA, também, receber informações sobre estudos a serem realizados no Brasil com medicamentos e outros produtos aqui desenvolvidos. Nesse sentido, a Resolução da Diretoria Colegiada (RDC) n $n^{\circ}$ 36/03, que dispõe sobre o registro de medicamentos novos, prevê, nas suas medidas antecedentes de registro para produtos novos nacionais e para produtos novos importados que venham fazer estudo fase III no Brasil, a apresentação de protocolos de pesquisas clínicas. Particularmente nos casos de medicamentos novos nacionais, a ANVISA pode ter um papel orientador quanto aos aspectos metodológicos desses estudos, considerando a experiência de que deficiências nesse ponto têm sido um grande fator limitante ao registro desse tipo de produto nessa Agência.

Para que a ANVISA possa ter um papel mais efetivo no controle dos ensaios clínicos com medicamentos e produtos para a saúde no Brasil, e particularmente no caso de medicamentos cujas informações geradas na fase de pesquisa possam ser utilizadas no momento do seu registro, a GEPEC está em processo de construção de novos regulamentos, que serão discutidos a seguir. Parte desses regulamentos já está em fase final de desenvolvimento, prevendo-se sua aprovação e publicação em 2004, enquanto que outros ainda estão sendo elaborados, prevendose sua implementação para um pouco mais tarde. Todos esses regulamentos, que eventualmente serão publicados em forma de RDC, normativas equivalentes às Portarias do Ministério da Saúde, passam por um período de consulta pública no qual qualquer pessoa pode apresentar a sua contribuição, criticando uma versão preliminar do documento. Concomitantemente, são feitas discussões diretamente com o setor regulado, de forma que todos os interessados têm a oportunidade de ser ouvidos antes da publicação das resoluções.

Para a área de pesquisa clínica, três propostas de RDC já passaram pela fase de consulta pública, foram aprovadas pela Diretoria Colegiada e, no momento em que este artigo está sendo redigido (junho de 2004), aguardam publicação. São elas: regulamento para elaboração de dossiê para a obtenção de Comunicado Especial para realização de pesquisa clínica (revisão da Portaria n 91//98), monitoramento de eventos adversos observados durante ensaios clínicos, e regulação das chamadas Organizações Representativas de Pesquisa Clínica (ORPCs), conhecidas pela sigla CRO (do inglês contract research organization). A revisão da Portaria $n^{\circ}$ 9l। pretende tornar a aprovação de ensaios clínicos pela ANVISA menos burocrática, além de introduzir alguns elementos de boas práticas clínicas. Outra inovação é que por esta proposta de RDC todos os ensaios clínicos realizados no país devem ser submetidos à ANVISA, e não apenas aqueles que necessitem de autorização para importação do produto. Já a proposta de RDC sobre eventos adversos torna obrigatória a notificação para a ANVISA daqueles ocorridos em ensaios clínicos conduzidos no Brasil, nacionais ou internacionais, com prazos diferentes para eventos de diferentes gravidades. A proposta sobre as ORPCs basicamente reconhece a existência de tais entidades, que representam os patrocinadores dos estudos em diversas funções a eles relacionadas, e define as suas responsabilidades e as dos patrocinadores por eles representados perante os sujeitos de pesquisa.

Está prevista para ser encaminhada para consulta pública, possivelmente em 2004, mais uma proposta de RDC, a qual diz respeito a inspeções ou auditorias de ensaios clínicos. Tais inspeções/auditorias, que terão como foco as boas práticas clínicas, poderão ser realizadas antes, durante ou após os estudos, e os estudos a elas submetidos poderão ser escolhidos ao acaso ou arbitrariamente, pelo tipo de intervenção ou de população estudadas. Averiguação de denúncias pode também ser um motivo para a sua realização. Na construção de tal proposta de RDC será imprescindível um bom entendimento entre ANVISA e CONEP, tendo em vista que as inspeções podem e devem avaliar aspectos éticos da condução dos estudos como, por exemplo, os relacionados à forma de obtenção, pelos investigadores, das assinaturas dos termos de consentimento livre e esclarecido dos sujeitos de pesquisa.

Paralelamente ao desenvolvimento de novas propostas de legislação, ampliando o controle da ANVISA sobre a pesquisa clínica no Brasil, a GEPEC também se preocupa em procurar atender a uma das grandes reivindicações das indústrias farmacêuticas e das ORPCs, que é o da demora em autorizar a realização do estudo (que na prática consiste em concessão de CE para emissão de LI), o que tem várias vezes impedido que ensaios clínicos multicêntricos financiados pelas indústrias sejam realizados no Brasil, já que tais estudos são competitivos, e outros países têm um tempo de aprovação menor que o do nosso. No que cabe à ANVISA, parte dessa demora está relacionada ao seu funcionamento, por vezes deficiente no que diz respeito a encaminhamento interno de documentos; falta de padronização de análise, e burocratização excessiva do processo. Esses pontos têm sido reconhecidos e tentativas de solução vêm sendo postas em prática. A análise dos 
NISHIOKA SA ET AL.

processos dentro da ANVISA também vem sendo feita em paralelo com a da CONEP, mas a aprovação desta é condição sine qua non para a aprovação da realização dos estudos no país. A existência de dois fóruns independentes de aprovação de ensaios clínicos no Brasil, CEPs/ CONEP e ANVISA pode parecer, ou mesmo ser, de fato, um processo ineficiente. Historicamente, porém, o processo se desenvolveu assim, e é improvável que haja alguma modificação nesse sentido, já que CONEP e ANVISA têm mandatos diferentes e complementares no que diz respeito à pesquisa clínica, restando assim que haja uma integração entre elas para tornar mais eficiente o processo de avaliação de protocolos de pesquisa no país. Cabe lembrar que rapidez é apenas um dos componentes da avaliação de eficiência, e que não necessariamente tem a ver com qualidade.

\section{O papel da ANVISA na regulação da pesquisa clínica: futuro}

Uma vez aprovadas e colocadas em prática as novas RDCs sobre pesquisa clínica, a ANVISA se colocará em um novo patamar perante os ensaios clínicos realizados no país. Em primeiro lugar, pela revisão da Portaria n $0^{\circ}$ 91 I/98, a ANVISA terá oportunidade de receber informaç̃̃es sobre todos os ensaios clínicos com medicamentos e produtos para a saúde que aqui ocorrerem, sejam eles financiados ou não pela indústria farmacêutica, e envolvendo ou não importação desses produtos. Além disso, ela passará a poder acompanhar os ensaios clínicos, por meio do recebimento e processamento das notificações de eventos adversos, e pelas inspeções/auditorias. Poderá, assim, verificar como esses estudos estão sendo conduzidos, ao invés de apenas aprová-los, como faz hoje em dia, baseando-se somente nas informaçōes apresentadas no protocolo.

Este acompanhamento longitudinal dos ensaios clínicos permitirá uma aproximação entre pesquisa clínica e registro, que no entender da ANVISA deve progressivamente se tornar uma realidade no Brasil. Vale ressaltar que, com o passar do tempo, a implementaçãa dessas propostas exigirá uma adequação do número de funcionários da ANVISA e sua capacitação, permitindo que a aprovação de ensaios clínicos no país não tenha apenas o olhar da segurança dos sujeitos de pesquisa, mas também o de sua utilidade para oferecer evidências que respaldem o registro dos produtos investigados. Isto deve valer não apenas para medicamentos novos sintéticos e semi-sintéticos, que são registrados pela GEPEC, mas também para outros tipos de medicamentos novos, como biológicos e fitoterápicos, que na ANVISA são registrados, respectivamente, pela Unidade de Produtos Biológicos e Hemoderivados (UPBIH) e pela Gerência de Isentos, Fitoterápicos e Homeopáticos (GIFHO).

A evolução da ANVISA em sua capacidade de aprovar estudos, atividade que é complementar à dos CEPs e CONEP, e também de acompanhá-los de maneira análoga a que fazem outras agências reguladoras de maior tradição, deverá trazer benefícios para a pesquisa clínica no país. Por um lado, irá aumentar a credibilidade do país como local de realização de ensaios clínicos multicêntricos financiados pela indústria farmacêutica, mantendo para o país as vantagens enumeradas anteriormente. Por outro, incentivará que ensaios clínicos com produtos desenvolvidos pela indústria brasileira tenham nível de excelência compatível com o exigido para o seu posterior registro tanto na ANVISA quanto em qualquer outra agência reguladora, favorecendo a possibilidade de expansão da indústria local.
A harmonização internacional de boas práticas clínicas hoje é uma realidade não só nos países desenvolvidos, mas também se desenvolve em escala mundial, com apoio de órgãos como a Organização Mundial da Saúde. O Brasil faz parte ativa da Pan American Network on Drug Regulatory Harmonization, promovida pela Organização Pan-Americana de Saúde, que inclui um grupo que procura harmonizar boas práticas clínicas dentro do continente americano. Não é apenas desejável mas imprescindível que o país evolua em seu controle sobre a qualidade dos ensaios clínicos aqui realizados. Do ponto de vista da ANVISA, esse controle está de acordo com a sua missão ("Proteger e promover a saúde da população garantindo a segurança sanitária de produtos e serviços e participando da construção de seu acesso"). Quanto às indústrias farmacêuticas de capital nacional e internacional, universidades, centros de pesquisa, e aos médicos e outros profissionais envolvidos em pesquisa clínica, esse controle lhes será favorável, pois deverá aumentar a credibilidade do país. Todos, portanto, têm a ganhar com ele.

\section{Conflito de interesse: não há.}

\section{SUMMARY}

\section{ANVISA and Clinical research in BraZil}

Conduction of clinical trials with drugs in Brazil requires prior approval by Committees for Ethics in Research (CEPS) and, in certain cases, by the National Commission for Ethics in Research (CONEP). Approval by the National Health Surveillance Agency (ANVISA), through its Office for New Drugs, Research and Clinical Trials (GEPEC), is currently required only for drugs and medical devices manufactured in other countries and therefore need permission to be imported. This article briefly reviews the history of clinical research regulation in Brazil, then presents an overview of the current regulatory role of ANVISA and its future prospective. Major points discussed are the new forthcoming ANVISA regulations, withemphasis on adverse event notifications and inspections/audits on clinical trials and their impact on clinical research in Brazil, from the standpoint of the industry, university, contract research organizations, physicians and other health professionals. Itshould be stressed that to decide upon the licensing of new drugs ANVISA must obtain information from clinical trials. [Rev Assoc Med Bras 2006; 52(I): 60-2]

KEY wORDS: ANVISA. Clinical research. Clinical trial. Regulation.

\section{REFERÊNCIAS}

I. Freitas CBD, Hossne WS. Pesquisa com seres humanos. In: Campana $A O$, organizador. Investigação científica na área médica. São Paulo: Manole; 2001 . p.205-19.

2. Lousana G, Acceturi C. Histórico da pesquisa clínica. In: Lousana G, organizador. Pesquisa clínica no Brasil. Rio de Janeiro: Revinter; 2002. p. -18.

Artigo recebido: 30/06/04

Aceito para publicação: 03/09/04 